\title{
Tackling blood culture contamination rates in the acute setting
}

\author{
Authors: Nicola West and Syed Haydar
}

\section{Aims}

Contaminated blood cultures cause a diagnostic and therapeutic challenge for microbiologists and physicians. There is potential risk to patients and expense to hospital trusts from inappropriate antibiotic administration and further tests. Data collection by the director of infection prevention and control (DIPC) found high contamination rates for blood culture sampling in the emergency department (ED) and emergency assessment unit (EAU). From August 2013 to January 2014, ED contamination rates were $6.7 \%$, compared with an acceptable $2 \%$ for the medical and surgical wards.

This project was undertaken to identify the factors causing this unprecedented increased contamination rate in the acute setting. The objective was to implement changes based on these findings that would reduce contamination rates and thus increase diagnostic precision, save money and improve the accuracy of patient treatment.

\section{Methods}

Notes of 50 patients known to have contaminated blood cultures were audited against local guidelines for whether sampling was performed and documented correctly in accordance with local guidelines. Groups of staff with high rates of contaminant sampling were identified.

Contamination data were collected for 6 months following changes made, and the results analysed for any reduction in contamination rates.

\section{Results}

Gaps in training were identified, and human factors in technique and beliefs were addressed. We established re-education of non-medical staff members who take blood cultures, advised increased accountability, and introduced changes to how doctors working in the acute setting will be trained.

Data collected for contamination levels since the changes were implemented is ongoing from April to August 2014. To date, a $1 \%$ reduction in contamination has been achieved.

\section{Conclusions}

Reducing blood culture contaminants means clearer decision making in cases of potential infection. Fewer patients will be subjected to unnecessary antibiotic treatment, thus reducing both the potential for antibiotic resistance and the financial implications from actively treating these patients.

\section{Conflict of interest statement}

We have no conflicts of interests to declare.

Authors: Broomfield Hospital, Mid Essex Hospitals NHS Trust, Chelmsford, UK 